\title{
Compressibility effects in a turbulent transport of temperature field
}

\author{
I. Rogachevskit and N. Kleeorin丹 \\ Department of Mechanical Engineering, Ben-Gurion University of the Negev, P. O. B. 653, Beer-Sheva 8410530, Israel \\ Nordita, Stockholm University and KTH Royal Institute of Technology, 10691 Stockholm, Sweden
}

(Dated: December 22, 2020)

\begin{abstract}
Compressibility effects in a turbulent transport of temperature field are investigated applying the quasi-linear approach for small Péclet numbers and the spectral $\tau$ approach for large Péclet numbers. Compressibility of a fluid flow reduces the turbulent diffusivity of the mean temperature field similarly to that for particle number density and magnetic field. However, expressions for the turbulent diffusion coefficient for the mean temperature field in a compressible turbulence are different from those for the mean particle number density and the mean magnetic field. Combined effect of compressibility and inhomogeneity of turbulence causes an increase of the mean temperature in the regions with more intense velocity fluctuations due to a turbulent pumping. Formally, this effect is similar to a phenomenon of compressible turbophoresis found previously [J. Plasma Phys. 84, 735840502 (2018)] for non-inertial particles or gaseous admixtures. Gradient of the mean fluid pressure results in an additional turbulent pumping of the mean temperature field. The latter effect is similar to turbulent barodiffusion of particles and gaseous admixtures. Compressibility of a fluid flow also causes a turbulent cooling of the surrounding fluid due to an additional sink term in the equation for the mean temperature field. There is no analog of this effect for particles.
\end{abstract}

\section{INTRODUCTION}

Compressibility of a fluid flow affects turbulent transport of particles, temperature and magnetic fields (see, e.g., [1 [5]), e.g., it causes qualitative changes in the properties of both, mean fields and fluctuations. Large-scale effects of turbulence on particle concentrations and temperature field are described by means of turbulent flux of particles and turbulent heat flux, respectively. For incompressible flow, main contribution to the turbulent fluxes is determined by turbulent diffusion of particles and temperature field. This corresponds to gradient turbulent transport of particles and temperature fields, e.g., the turbulent flux of particles is directed opposite to gradient of the mean particle number density, while the turbulent heat flux is directed opposite to gradient of the mean fluid temperature.

Compressibility of a turbulent flow results in a reduction of turbulent diffusivity of a mean particle number density at small [6] and large [5, 7] Péclet numbers. The Péclet number is the ratio of nonlinear to diffusion terms in the equation for particle number density fluctuations. Similar effect of the reduction of turbulent magnetic diffusivity by compressible turbulence exists also for the mean magnetic field at small [6, 8] and large 5, 7] magnetic Reynolds numbers. The conclusion about the reduction of turbulent diffusivity by the compressibility of fluid flow has been also confirmed by the test-field method in direct numerical simulations for an irrotational homogeneous deterministic flow [6]. Various aspects related to compressibility effects on turbulent transport have been studied using different analytical approaches (see for a review, [5]), e.g., the quasi-

\footnotetext{
* gary@bgu.ac.il http://www.bgu.ac.il/ gary

$\dagger$ nat@bgu.ac.il
}

linear approach [6 8], the spectral tau approach [7], the path-integral approach [9 11], the multiple-scale directinteraction approximation [12, 13], etc.

Compressibility of a turbulent flow causes additional non-gradient contribution to turbulent flux of particles that is proportional to a product of the mean particle number density and effective pumping velocity. In a density stratified turbulence, the effective pumping velocity of particles is proportional to the gradient of the mean fluid density multiplied by turbulent diffusion coefficient [10, 11]. The pumping effect results in accumulation of particles in regions of maximum mean fluid density.

In a temperature stratified turbulence, similar effect referred as turbulent thermal diffusion results in a turbulent non-diffusive flux of particles in the direction of the turbulent heat flux, so that particles are accumulated in the vicinity of the mean temperature minimum 10, 11]. This phenomenon has been studied theoretically 14 18], found in direct numerical simulations 7, 19, 20], detected in different laboratory experiments [18, 21 23], and atmospheric turbulence with temperature inversions 24. This effect has been shown to be important for concentrating dust in protoplanetary discs [25]. Density stratification which causes turbulent pumping of particles, becomes weaker with increasing compressibility, i.e., with increasing the Mach number [7].

Compressibility of a fluid flow in inhomogeneous turbulence also results in a new pumping effect of particles from regions of low to high turbulent intensity both for small and large Péclet numbers. This effect has been interpreted in [7] as a compressible turbophoresis of noninertial particles and gaseous admixtures, while the classical turbophoresis effect for incompressible inhomogeneous turbulence [26 30] exists only for inertial particles and causes them to be pumped to regions with lower turbulent intensity.

The compressibility of a turbulent fluid flow affects also 
passive scalar fluctuations. In particular, it results in a slow scale-dependent turbulent diffusion of a small-scale passive scalar fluctuations for large Pèclet numbers [9]. In addition, the level of the passive scalar fluctuations in the presence of a gradient of the mean passive scalar field in compressible turbulent flow can be fairly strong. On the other hand, passive scalar transport in a density stratified turbulent fluid flow is accompanied by formation of largescale structures due to instability of the mean passive scalar field in inhomogeneous turbulent velocity field [9].

Another interesting feature for a compressible temperature stratified turbulence is that turbulent flux of entropy is different from turbulent convective flux of fluid internal energy 31, 32]. In particular, in a low-Machnumber approximation as well as in the framework of the mean-field approach, turbulent flux of entropy is given by $\boldsymbol{F}_{s}=\bar{\rho}\left\langle s^{\prime} \boldsymbol{u}\right\rangle$, where $\bar{\rho}$ is the mean fluid density and $s^{\prime}$ and $\boldsymbol{u}$ are fluctuations of entropy and velocity, respectively, and the angular brackets $\langle\ldots\rangle$ denote ensemble averaging. On the other hand, the turbulent convective flux of the fluid internal energy is $\boldsymbol{F}_{c}=\bar{T} \bar{\rho}\left\langle s^{\prime} \boldsymbol{u}\right\rangle$, where $\bar{T}$ is the mean fluid temperature. This turbulent convective flux is well-known in the astrophysical and geophysical literature, and it cannot be used as a turbulent flux in the equation for the mean entropy. This is exact result for low-Mach-number temperature stratified turbulence and is independent of the turbulence model used [32].

Temperature fluctuations and anomalous scaling in a low-Mach-number compressible turbulent flow have been studied in [33]. Due to compressibility and external pressure fluctuations, the anomalous scaling (i.e., the violation of the dimensional analysis predictions for the scaling laws) may occur in the second moment of the temperature field. The cause of the anomalous behavior is a compressibility-induced depletion of the turbulent diffusion of the second moment of the temperature field [33].

In spite of many studies of turbulent transport of passive scalar, some large-scale (mean-field) features related to compressibility effects on turbulent transport of temperature field are not known. In the present paper, we study compressibility effects in turbulent transport of the mean temperature field, i.e., we consider here mean-field effects. This paper is organized as follows. In Section II we outline the governing equations. Turbulent heat flux and level of temperature fluctuations are determined for small Péclet numbers in Section III and for large Péclet numbers in Section IV. In Sections III-IV we also outline the method of derivations and approximations made for study of the compressibility effects. In Section V we discuss how a homogeneous compressible turbulence can cause a turbulent cooling of the surrounding fluid. Finally, conclusions are drawn in Section VI. In Appendix A we outline the multi-scale approach used in the present study. Details of the derivation of turbulent heat flux and level of temperature fluctuations are given in Appendix B for small Péclet numbers and in Appendix C for large Péclet numbers.

\section{GOVERNING EQUATIONS}

Evolution of temperature field $T(t, \boldsymbol{r})$ in a compressible fluid velocity field $\mathbf{U}(t, \boldsymbol{r})$ is given by [34]

$$
\frac{\partial T}{\partial t}+(\mathbf{U} \cdot \boldsymbol{\nabla}) T+(\gamma-1) T(\boldsymbol{\nabla} \cdot \boldsymbol{U})=D \Delta T+J_{\nu},
$$

where $D$ is the molecular thermal conductivity, $\gamma=c_{\mathrm{p}} / c_{\mathrm{v}}$ is the ratio of specific heats and $J_{\nu}$ is the heating source caused, e.g., by a viscous dissipation.

In a compressible flow, Eq. (10) for the temperature field is different from equation for particle number density $n(t, r)$ 35, 36]

$$
\frac{\partial n}{\partial t}+\nabla \cdot(n \boldsymbol{U})=D_{n} \Delta n
$$

where $D_{n}$ is the coefficient of Brownian (molecular) diffusion of particles.

We consider a compressible turbulent flow when Mach number can be not small. To derive equations for the turbulent heat flux and the level of temperature fluctuations, we apply the mean-field approach. In particular, the fluid temperature, pressure, density and velocity are decomposed into mean and fluctuating parts, where the fluctuating parts have zero mean values, i.e., the Reynolds averaging is applied here, which easily separates fluctuations from mean fields. For example, the density-weighted averaging quantities [37, 38] are usually difficult to extract from laboratory and atmospheric measurements or from astrophysical observations.

In the framework of the mean-field approach, the fluid temperature is $T=\bar{T}+\theta$, the fluid pressure is $P=\bar{P}+p$ and the fluid density is $\rho=\underline{\bar{\rho}}+\rho^{\prime}$, where $\bar{T}=\langle T\rangle$ is the mean fluid temperature, $\bar{P}=\langle P\rangle$ is the mean fluid pressure and $\bar{\rho}=\langle\rho\rangle$ is the mean fluid density, $\theta$ are temperature fluctuations, $p$ are pressure fluctuations and $\rho^{\prime}$ are density fluctuations. The angular brackets denote an ensemble averaging. Similarly, $\boldsymbol{U}=\overline{\boldsymbol{U}}+\boldsymbol{u}$, where $\overline{\boldsymbol{U}}=\langle\boldsymbol{U}\rangle$ is the mean fluid velocity, and $\boldsymbol{u}$ are velocity fluctuations. For simplicity, we consider the case $\overline{\boldsymbol{U}}=0$.

Averaging Eq. (1) over ensemble of turbulent velocity field, we arrive at equation for the mean temperature field as

$$
\frac{\partial \bar{T}}{\partial t}+\nabla \cdot\langle\theta \boldsymbol{u}\rangle=-(\gamma-2)\langle\theta(\boldsymbol{\nabla} \cdot \boldsymbol{u})\rangle+D \Delta \bar{T}+\bar{J}_{\nu},
$$

where $\boldsymbol{F}=\langle\theta \boldsymbol{u}\rangle$ is the turbulent heat flux, $\bar{J}_{\nu}$ is the mean heating source caused by the viscous dissipation of the turbulent kinetic energy, and $I_{\mathrm{S}}=-(\gamma-2)\langle\theta(\boldsymbol{\nabla} \cdot \boldsymbol{u})\rangle$ is the mean sink term resulting in a turbulent cooling due to compressibility effects (see Section V). Using Eqs. (1) and (3), we obtain equation for temperature fluctuations, $\theta(\boldsymbol{x}, t)=T-\bar{T}$ :

$$
\frac{\partial \theta}{\partial t}+\mathcal{Q}-D \boldsymbol{\nabla} \theta=-(\boldsymbol{u} \cdot \boldsymbol{\nabla}) \bar{T}-(\gamma-1) \bar{T} \boldsymbol{\nabla} \cdot \boldsymbol{u},
$$


where

$$
\mathcal{Q}=\boldsymbol{\nabla} \cdot(\theta \boldsymbol{u}-\langle\boldsymbol{u} \theta\rangle)+(\gamma-2)[\theta \boldsymbol{\nabla} \cdot \boldsymbol{u}-\langle\theta \boldsymbol{\nabla} \cdot \boldsymbol{u}\rangle]
$$

are nonlinear terms and

$$
I=-(\boldsymbol{u} \cdot \boldsymbol{\nabla}) \bar{T}-(\gamma-1) \bar{T} \boldsymbol{\nabla} \cdot \boldsymbol{u}
$$

are the source terms of temperature fluctuations. The ratio of the nonlinear term to the diffusion term is the Péclet number, that is estimated as $\mathrm{Pe}=u_{0} \ell_{0} / D$, where $u_{0}$ is the characteristic turbulent velocity in the integral (energy-containing) scale $\ell_{0}$ of turbulence. We consider a one way coupling, i.e., we take into account the effect of turbulence on the temperature field, but neglect the feedback effect of the temperature on the turbulence.

To determine the turbulent heat flux and the level of temperature fluctuations, and to take into account smallscale properties of the turbulence, we use two-point correlation functions. For fully developed turbulence, scalings for the turbulent correlation time and the turbulent kinetic energy spectrum are related via the Kolmogorov scalings [1, 2, 39 41]. We consider the cases with small and large Péclet and Reynolds numbers.

In the framework of the mean-field approach, we assume that there is a separation of spatial and temporal scales, i.e., $\ell_{0} \ll L_{T}$ and $\tau_{0} \ll t_{T}$, where $L_{T}$ and $t_{T}$ are the characteristic spatial and temporal scales characterizing the variations of the mean temperature field and $\tau_{0}=\ell_{0} / u_{0}$. The mean fields depend on "slow" variables, while fluctuations depend on "fast" variables. Separation into slow and fast variables is widely used in theoretical physics, and all calculations are reduced to the Taylor expansions of all functions using small parameters $\ell_{0} / L_{T}$ and $\tau_{0} / t_{T}$. The findings are further truncated to leading order terms. Separation to slow and fast variables is performed by means of a standard multi-scale approach [42] discussed in details in Appendix A.

\section{TURBULENT HEAT FLUX AND LEVEL OF TEMPERATURE FLUCTUATIONS FOR SMALL PÉCLET NUMBERS}

In this section we derive equations for the turbulent heat flux and the level of temperature fluctuations for small Péclet numbers using the quasi-linear approach. For a random flow with small Péclet and Reynolds numbers, there are no universal scalings for the correlation time and the turbulent kinetic energy spectrum. This is the reason why we use non-instantaneous two-point correlation functions in this case. In the framework of the quasi-linear approach, we neglect the nonlinear term $\mathcal{Q}$, but keep the molecular diffusion term in Eq. (4). We rewrite this equation in Fourier space and find the solution of this equation, given by Eq. (B1) in Appendix B. Using this solution and applying the multi-scale approach (see [42] and Appendix A), we arrive at expressions for the turbulent heat flux and the level of temperature fluctuations in Fourier space for small Péclet numbers as

$$
\begin{aligned}
\left\langle\theta u_{j}\right\rangle= & -\frac{\gamma-1}{2}\left[\bar{T} \int G_{D}\left(\nabla_{i}-2 D k^{2} G_{D} k_{i m} \nabla_{m}+2 i k_{i}\right) f_{i j} d \boldsymbol{k} d \omega\right. \\
& \left.-\left(\nabla_{i} \bar{T}\right) \int G_{D}\left(\frac{\gamma-3}{\gamma-1} \delta_{i m}+2 D k^{2} G_{D} k_{i m}+k_{m} \frac{\partial}{\partial k_{i}}\right) f_{m j}\right] d \boldsymbol{k} d \omega, \\
\left\langle\theta^{2}\right\rangle= & \frac{\gamma-1}{4}\left\{\bar{T} \int G_{D}\left[\left(2 D k^{2} G_{D} k_{j n} \nabla_{n}-\nabla_{j}\right) F_{j}^{(+)}+2 i k_{j} F_{j}^{(-)}\right] d \boldsymbol{k} d \omega\right. \\
& \left.+\left(\nabla_{n} \bar{T}\right) \int G_{D}\left[\frac{\gamma-3}{\gamma-1} \delta_{j n}+2 D k^{2} G_{D} k_{j n}+k_{j} \frac{\partial}{\partial k_{n}}\right] F_{j}^{(+)}\right\} d \boldsymbol{k} d \omega .
\end{aligned}
$$

Details of derivations of Eqs. (5) and (6) are given in Appendix B. Here $G_{D} \equiv G_{D}(\boldsymbol{k}, \omega)=\left(D \boldsymbol{k}^{2}+i \omega\right)^{-1}, f_{i j} \equiv$ $f_{i j}(\boldsymbol{k}, \omega)=\left\langle u_{i}(\boldsymbol{k}, \omega) u_{j}(-\boldsymbol{k},-\omega)\right\rangle, F_{j}^{( \pm)}=F_{j}(\boldsymbol{k}, \omega) \pm$ $F_{j}(-\boldsymbol{k}, \omega)$, where $F_{j}(\boldsymbol{k}, \omega)=\left\langle\theta(\boldsymbol{k}, \omega) u_{j}(-\boldsymbol{k},-\omega)\right\rangle$ is the turbulent heat flux in Fourier space, $\delta_{i j}$ is the Kronecker unit tensor and $k_{i j}=k_{i} k_{j} / k^{2}$. Since we consider a one way coupling, the correlation function $f_{i j}$ in Eqs. (5) and (6) should be replaced by $f_{i j}^{(0)}$ for the background random flow with zero turbulent heat flux.

We use a statistically stationary, density-stratified, inhomogeneous, compressible and non-helical background random flow determined by the following correlation function in Fourier space [5, 7]:

$$
\begin{aligned}
f_{i j}^{(0)}(\boldsymbol{k}, \omega)= & \frac{\Phi(\omega)}{8 \pi k^{2}\left(1+\sigma_{c}\right)}\left\{E(k)\left[\delta_{i j}-k_{i j}+\frac{i}{k^{2}}\left(k_{j} \lambda_{i}-k_{i} \lambda_{j}\right)+\frac{i}{2 k^{2}}\left(k_{i} \nabla_{j}-k_{j} \nabla_{i}\right)\right]\right. \\
& \left.+2 \sigma_{c} E_{c}(k)\left[k_{i j}+\frac{i}{2 k^{2}}\left(k_{i} \nabla_{j}-k_{j} \nabla_{i}\right)\right]\right\}\left\langle\boldsymbol{u}^{2}\right\rangle,
\end{aligned}
$$


where $\boldsymbol{\lambda}=-\boldsymbol{\nabla} \ln \bar{\rho}$ characterizes the fluid density stratification, $\sqrt{\left\langle\boldsymbol{u}^{2}\right\rangle}$ is the characteristic turbulent velocity at the maximum scale $\ell_{0}$ of random motions, and the parameter

$$
\sigma_{c}=\frac{\left\langle(\nabla \cdot \boldsymbol{u})^{2}\right\rangle}{\left\langle(\nabla \times \boldsymbol{u})^{2}\right\rangle}
$$

is the degree of compressibility of the turbulent velocity field. We considered a weakly anisotropic background turbulence. In particular, in derivation of Eq. (7), we assumed that $\ell_{0} \ll H_{\rho}$ and $\ell_{0} \ll L_{u}$, where $L_{u}=$ $\left|\nabla \ln \left\langle\boldsymbol{u}^{2}\right\rangle\right|^{-1}$ is the characteristic scale of the inhomogeneity of turbulence, and $H_{\rho}=|\boldsymbol{\lambda}|^{-1}=|\boldsymbol{\nabla} \ln \bar{\rho}|^{-1}$ is the mean density stratification scale, which is assumed to be constant. These conditions allow us to take into account leading effects in Eq. (7), which are linear in stratification, $\propto|\boldsymbol{\lambda}|$, and inhomogeneity of turbulence, $\propto\left|\nabla \ln \left\langle\boldsymbol{u}^{2}\right\rangle\right|$. We neglect in Eq. (77) high-order effects which are of the order of $\mathrm{O}\left(\lambda^{2}\left\langle\boldsymbol{u}^{2}\right\rangle\right), \mathrm{O}\left(\nabla^{2}\left\langle\boldsymbol{u}^{2}\right\rangle\right)$, $\mathrm{O}\left(\lambda_{i} \nabla_{i}\left\langle\boldsymbol{u}^{2}\right\rangle\right)$.

Generally, stratification also contributes to $\operatorname{div} \boldsymbol{u}$, i.e., it contributes to the parameter $\sigma_{c}$. Since this contribution is small, i.e., it is of the order of $\sim \mathrm{O}\left(\lambda^{2}\left\langle\boldsymbol{u}^{2}\right\rangle\right)$, we neglect this contribution in Eq. (7). This allows us to separate effects of the arbitrary Mach number, characterized by the parameter $\sigma_{c}$, and density stratification, described by $\boldsymbol{\lambda}$. The degree of compressibility $\sigma_{c}$ depends on the Mach number. This dependence is not known for arbitrary Mach numbers and can be determined, e.g., in direct numerical simulations.

In Eq. (7), $E(k)$ and $E_{c}(k)$ are the spectrum functions for incompressible and compressible parts of a random flow. We assume that the random flow have a power-law spectrum for incompressible $E(k)=(q-1)\left(k / k_{0}\right)^{-q} k_{0}^{-1}$ and compressible $E_{c}(k)=\left(q_{c}-1\right)\left(k / k_{0}\right)^{-q_{c}} k_{0}^{-1}$ parts, where the wave number varies in the range, $k_{0} \leq k \leq k_{\nu}$. Here $k_{\nu}=1 / \ell_{\nu}$ is the wave number based on the viscous scale $\ell_{\nu}$, and $k_{0}=1 / \ell_{0} \ll k_{\nu}$. We assume also that there are no random motions for $k<k_{0}$. In the model of a compressible background turbulence used in 7], the exponents $q=q_{c}$. In the present study, we consider the case when the spectrum exponents of the incompressible and compressible parts of random motions are different, i.e., $q \neq q_{c}$.

We assume that the frequency function $\Phi(\omega)$ has a Lorentz profile, $\Phi(\omega)=\left[\pi \tau_{0}\left(\omega^{2}+\tau_{0}^{-2}\right)\right]^{-1}$, which corresponds to the correlation function $\left\langle u_{i}(t) u_{j}(t+\tau)\right\rangle \propto$ $\exp \left(-\tau / \tau_{0}\right)$. Here the correlation time for small Péclet numbers $\tau_{0} \equiv \ell_{0} / u_{0} \gg\left(D k^{2}\right)^{-1}$ for all turbulent scales. To derive Eq. (7) we use identities given in Appendix B. Different contributions to Eq. (7) have been discussed in [5, 7, 9, 43].

Integration in $\omega$ and $\boldsymbol{k}$ space in Eq. (5) yields an equation for the turbulent heat flux for small Péclet numbers:

$$
\langle\theta \boldsymbol{u}\rangle=\bar{T} \boldsymbol{V}^{\mathrm{eff}}-D_{T} \nabla \bar{T},
$$

where the turbulent diffusivity $D_{T}$ and the effective pumping velocity $\boldsymbol{V}^{\text {eff }}$ are given by

$$
D_{T}=\frac{(q-1)}{3(q+1)} \frac{\tau_{0}\left\langle\boldsymbol{u}^{2}\right\rangle}{1+\sigma_{c}} \operatorname{Pe}\left[\gamma-\frac{1}{2}(3 \gamma-5) \sigma_{c} C_{\sigma}\right],
$$

$$
\boldsymbol{V}^{\mathrm{eff}}=(\gamma-1) \frac{(q-1)}{3(q+1)} \frac{\tau_{0}\left\langle\boldsymbol{u}^{2}\right\rangle}{\left(1+\sigma_{c}\right)} \operatorname{Pe}\left[\frac{3}{2} C_{\sigma} \sigma_{c} \boldsymbol{\lambda}_{u}+\boldsymbol{\lambda}_{P}\right]
$$

Here $\boldsymbol{\lambda}_{u}=\nabla \ln \left\langle\boldsymbol{u}^{2}\right\rangle, \boldsymbol{\lambda}_{P}=\nabla \ln \bar{P}$, and

$$
C_{\sigma}=\frac{\left(q_{c}-1\right)(q+1)}{\left(q_{c}+1\right)(q-1)} .
$$

We take into account that the equation of state for ideal gas yields $\boldsymbol{\lambda}=-\boldsymbol{\lambda}_{P}+\boldsymbol{\nabla} \ln \bar{T}$. Since $\tau_{0} \mathrm{Pe}=\ell_{0}^{2} / D$, the turbulent transport coefficients given by Eqs. (10) and (11) are determined only by the microphysical diffusion time scale $\ell_{0}^{2} / D$ for small Péclet numbers. Equation (10) implies that for small Péclet numbers, compressibility effects in most of the cases decrease the turbulent diffusivity. Indeed, for $\gamma \geq 5 / 3$, the derivative $\partial D_{T} / \partial \sigma_{c}$ is always negative, i.e., the compressibility effects decrease the turbulent diffusivity. When $1<\gamma<5 / 3$, the derivative $\partial D_{T} / \partial \sigma_{c}$ is negative when $C_{\sigma}<2 \gamma /(5-3 \gamma)$. For example, for $q=q_{c}$ the derivative $\partial D_{T} / \partial \sigma_{c}$ is always negative. When $q=5 / 3$ (i.e., for the Kolmogorov spectrum) and $q_{c}=2$ (i.e., for the Burgers turbulence with shock waves), the derivative $\partial D_{T} / \partial \sigma_{c}$ is negative when $10 / 9<\gamma<5 / 3$. Note that, the total diffusivity $D+D_{T}$ cannot be negative, because for $\mathrm{Pe} \ll 1$ the molecular diffusivity is much larger than the turbulent one, $D \gg\left|D_{T}\right|$.

The first term $\left(\propto \sigma_{c} \boldsymbol{\nabla}\left\langle\boldsymbol{u}^{2}\right\rangle\right)$ in Eq. (11) for the effective pumping velocity $\boldsymbol{V}^{\text {eff }}$ of the mean temperature field describes a combined effect of compressibility of fluid flow and inhomogeneity of turbulence. This effect increases the mean temperature field in the regions with more intense velocity fluctuations due to turbulent pumping. This effect is similar to a phenomenon of compressible turbophoresis found previously for non-inertial particles or gaseous admixtures [7].

The second term $\left(\propto \boldsymbol{\lambda}_{P}\right)$ in Eq. (11) describes an additional turbulent pumping effect due to the gradient of the mean fluid pressure. This effect is similar to turbulent barodiffusion of particles and gaseous admixtures [11]. The physics of these effects is discussed in the next section. Note that the expressions for turbulent diffusion and the effective pumping velocity for the mean temperature field in a compressible turbulence are different from those for particle number density and magnetic field (see [7]), because equations for particle number density or magnetic field are different from those for the fluid temperature (see discussion at the end of Section IV).

Integration in $\omega$ and $\boldsymbol{k}$ space in Eq. (6) yields the expression for the level of temperature fluctuations for small Péclet numbers as 


$$
\begin{aligned}
\left\langle\theta^{2}\right\rangle= & (\gamma-1)^{2}\left(\frac{q_{c}-1}{q_{c}+1}\right)\left(\frac{\sigma_{c}}{1+\sigma_{c}}\right) \operatorname{Pe}^{2} \bar{T}^{2} \\
& +\frac{q-1}{3(q+3)} \operatorname{Pe}^{2} \ell_{0}^{2}\left\{(\boldsymbol{\nabla} \bar{T})^{2}+\frac{1}{8}(\gamma-1)\left[6(\boldsymbol{\lambda} \cdot \boldsymbol{\nabla})+(\gamma+3)\left(\boldsymbol{\lambda}_{u} \cdot \boldsymbol{\nabla}\right)\right] \bar{T}^{2}\right\} .
\end{aligned}
$$

The first term in the right hand side of Eq. (13) determines a dominant contribution of the compressible part of velocity fluctuations to the level of temperature fluctuations. Here we neglect much smaller contributions $\sim \mathrm{O}\left[\ell_{0}^{2} /\left(L_{T} L_{u}\right)\right], \mathrm{O}\left[\ell_{0}^{2} /\left(L_{T} H_{\rho}\right)\right], \mathrm{O}\left[\ell_{0}^{2} / L_{T}^{2}\right]$, caused by the compressible part of velocity fluctuations, where $L_{T}$ is the characteristic scale of the mean temperature field variations. For small $\sigma_{c}$, the level of temperature fluctuations is determined by the terms given by the second line of Eq. (13) and caused by the mean temperature gradient and the density stratified and inhomogeneous part of velocity fluctuations.

\section{TURBULENT HEAT FLUX AND LEVEL OF TEMPERATURE FLUCTUATIONS FOR LARGE PÉCLET NUMBERS}

In this section we determine the turbulent heat flux and the level of temperature fluctuations for large Péclet and Reynolds numbers. We consider fully developed turbulence, where the Strouhal number is of the or- der of unity and the turbulent correlation time is scaledependent, so we apply the Fourier transformation only in $\boldsymbol{k}$ space.

The procedure of the derivations of the expressions for the turbulent heat flux and the level of temperature fluctuations includes: (i) derivation of equations for the second moments in $\boldsymbol{k}$ space using the multi-scale approach, (ii) application of the spectral $\tau$ approach (see below) which allows us to relate the deviations of the third moments (appearing due to nonlinear terms) from those of the background turbulence with the deviations of the second moments, (iii) solution of the equations for the second moments in the $\boldsymbol{k}$ space, and (iv) inverse transformation to the physical space to obtain formulas for the turbulent heat flux and the level of temperature fluctuations.

Starting with Eq. (4) for the temperature fluctuations $\theta$ and the Navier-Stokes equation for the velocity $\boldsymbol{u}$ written in Fourier space, we derive dynamic equations for the turbulent heat flux and level of temperature fluctuations as

$$
\begin{aligned}
& \frac{\partial F_{j}}{\partial t}=-\frac{1}{2}(\gamma-1)\left[\bar{T}\left(2 i k_{i}+\nabla_{i}\right) f_{i j}-\left(\nabla_{i} \bar{T}\right)\left(\frac{\gamma-3}{\gamma-1} \delta_{i m}+k_{m} \frac{\partial}{\partial k_{i}}\right) f_{m j}\right]+\hat{\mathcal{M}} F_{j}^{(\mathrm{III})}, \\
& \frac{\partial E_{\theta}}{\partial t}=\frac{1}{2}(\gamma-1)\left[\bar{T}\left(2 i k_{j} F_{j}^{(-)}-\nabla_{j} F_{j}^{(+)}\right)+\left(\nabla_{m} \bar{T}\right)\left(k_{j} \frac{\partial}{\partial k_{m}}+\frac{\gamma-3}{\gamma-1} \delta_{j m}\right) F_{j}^{(+)}\right]+\hat{\mathcal{M}} E_{\theta}^{(\mathrm{III})} .
\end{aligned}
$$

Details of derivations of Eqs. (14)-15) are given in Appendix C. Here $F_{j}(\boldsymbol{k})=\left\langle\theta(\boldsymbol{k}) u_{j}(-\boldsymbol{k})\right\rangle, E_{\theta}(\boldsymbol{k})=$ $\langle\theta(\boldsymbol{k}) \theta(-\boldsymbol{k})\rangle, \quad f_{i j}(\boldsymbol{k})=\left\langle u_{i}(\boldsymbol{k}) u_{j}(-\boldsymbol{k})\right\rangle$, and $F_{j}^{( \pm)}=$ $F_{j}(\boldsymbol{k}) \pm F_{j}(-\boldsymbol{k})$, the third-order moment terms $\hat{\mathcal{M}} F_{j}^{(\mathrm{III})}$ and $\hat{\mathcal{M}} E_{\theta}^{(\mathrm{III})}$ written in $\boldsymbol{k}$ space and appearing due to the nonlinear terms are given by Eqs. (C8) and (C9) in Appendix C.

Equations (14) and (15) for the second moment include first-order spatial differential operators $\hat{\mathcal{M}}$ applied to the third-order moments $F^{(\mathrm{III})}$. The problem arises how to close Eqs. (14) and (15), i.e., how to express the third-order terms $\hat{\mathcal{M}} F^{(\mathrm{III})}$ through the lower moments [1, 2, 39, 44]. We use the spectral $\tau$ approach which is a universal tool in turbulent transport for strongly nonlinear systems. The spectral $\tau$ approximation postulates that the deviations of the third-moment terms, $\hat{\mathcal{M}} F^{(\mathrm{III})}(\boldsymbol{k})$, from the contributions to these terms af- forded by the background turbulence, $\hat{\mathcal{M}} F^{(\mathrm{III}, 0)}(\boldsymbol{k})$, can be expressed through similar deviations of the second moments, $F^{(\mathrm{II})}(\boldsymbol{k})-F^{(\mathrm{II}, 0)}(\boldsymbol{k})$ as

$$
\hat{\mathcal{M}} F^{(\mathrm{III})}(\boldsymbol{k})-\hat{\mathcal{M}} F^{(\mathrm{III}, 0)}(\boldsymbol{k})=-\frac{F^{(\mathrm{II})}(\boldsymbol{k})-F^{(\mathrm{II}, 0)}(\boldsymbol{k})}{\tau_{r}(k)},
$$

(see, e.g., 44 46]), where $\tau_{r}(k)$ is the scale-dependent relaxation time which can be identified with the correlation time $\tau(k)$ of the turbulent velocity field for large fluid Reynolds numbers and large Péclet numbers. Here functions with superscript (0) correspond to background turbulence with zero turbulent heat flux. Therefore, Eq. (16) is reduced to $\hat{\mathcal{M}} F_{i}^{(\mathrm{III})}(\boldsymbol{k})=-F_{i}(\boldsymbol{k}) / \tau(k)$ and $\hat{\mathcal{M}} E_{\theta}^{(\mathrm{III})}(\boldsymbol{k})=-E_{\theta}(\boldsymbol{k}) / \tau(k)$. Validation of the $\tau$ approximation for different situations has been performed in various numerical simulations [6, 7, 19, 47 [53]. We apply the $\tau$ approximation only to study the deviations from the 
background turbulence which are caused by the spatial derivatives of the mean temperature. The background compressible inhomogeneous and density stratified turbulence is assumed to be known (see below).

The $\tau$ approximation is a sort of the high-order closure and in general is similar to Eddy Damped Quasi Normal Markovian (EDQNM) approximation. However some principal difference exists between these two approaches [44, 45]. The EDQNM closures do not relax to equilibrium (the background turbulence), and the EDQNM approach does not describe properly the motions in the equilibrium state in contrast to the $\tau$ approximation. Within the EDQNM theory, there is no dynamically determined relaxation time, and no slightly perturbed steady state can be approached. In the $\tau$ approximation, the relaxation time for small departures from equilibrium is determined by the random motions in the equilibrium state, but not by the departure from the equilibrium. As follows from the analysis in [44], the $\tau$ approximation describes the relaxation to the equilibrium state (the background turbulence) much more accurately than the EDQNM approach.

Next, we assume that the characteristic times of variation of the second moments $F_{i}$ and $E_{\theta}$ are substantially larger than the correlation time $\tau(k)$ in all turbulence scales. This allows us to get steady-state solutions of Eqs. (14) and (15) as

$$
\begin{aligned}
& \left\langle\theta u_{j}\right\rangle=-\frac{1}{2}(\gamma-1) \int \tau(k)\left[\bar{T}\left(2 i k_{i}+\nabla_{i}\right) f_{i j}-\left(\nabla_{i} \bar{T}\right)\left(\frac{\gamma-3}{\gamma-1} \delta_{i m}+k_{m} \frac{\partial}{\partial k_{i}}\right) f_{m j}\right] d \boldsymbol{k}, \\
& \left\langle\theta^{2}\right\rangle=\frac{1}{2}(\gamma-1) \int \tau(k)\left[\bar{T}\left(2 i k_{j} F_{j}^{(-)}-\nabla_{j} F_{j}^{(+)}\right)+\left(\nabla_{m} \bar{T}\right)\left(k_{j} \frac{\partial}{\partial k_{m}}+\frac{\gamma-3}{\gamma-1} \delta_{j m}\right) F_{j}^{(+)}\right] d \boldsymbol{k} .
\end{aligned}
$$

In Eqs. (17) and (18) we take into account a one way coupling, i.e., we neglect the effect of the mean temperature gradients on the turbulent velocity field. This implies that we replace the correlation function $f_{i j}$ in Eqs. (17) and (18) by $f_{i j}^{(0)}$ for the background turbulent flow with zero turbulent heat flux.

We use statistically stationary, density-stratified, inhomogeneous, compressible and non-helical background turbulence, which is determined by the following correlation function in $\boldsymbol{k}$ space [5, 7]:

$$
\begin{aligned}
f_{i j}^{(0)}(\boldsymbol{k})= & \frac{1}{8 \pi k^{2}\left(1+\sigma_{c}\right)}\left\{E(k)\left[\delta_{i j}-k_{i j}+\frac{i}{k^{2}}\left(k_{j} \lambda_{i}-k_{i} \lambda_{j}\right)+\frac{i}{2 k^{2}}\left(k_{i} \nabla_{j}-k_{j} \nabla_{i}\right)\right]\right. \\
& \left.+2 \sigma_{c} E_{c}(k)\left[k_{i j}+\frac{i}{2 k^{2}}\left(k_{i} \nabla_{j}-k_{j} \nabla_{i}\right)\right]\right\}\left\langle\boldsymbol{u}^{2}\right\rangle .
\end{aligned}
$$

We assume here that the background turbulence is of Kolmogorov type with constant energy flux over the spectrum, i.e., the velocity fluctuations spectrum for the incompressible part of turbulence in the range of wave numbers $k_{0}<k<k_{\nu}$ is $E(k)=-d \bar{\tau}(k) / d k$, where the function $\bar{\tau}(k)=\left(k / k_{0}\right)^{1-q}$ with $1<q<3$ being the exponent of the turbulent kinetic energy spectrum. The condition $q>1$ corresponds to finite turbulent kinetic energy for very large fluid Reynolds numbers, while $q<3$ corresponds to finite dissipation of the turbulent kinetic energy at the viscous scale [see, e.g., 1, 2, 39 41]. Similarly, the turbulent kinetic energy spectrum for the compressible part of turbulence is $E_{c}(k)=-d \bar{\tau}_{c}(k) / d k$, where the function $\bar{\tau}_{c}(k)=\left(k / k_{0}\right)^{1-q_{c}}$ with $1<q_{c}<3$. For instance, the exponent of the incompressible part of the turbulent kinetic energy spectrum $q=5 / 3$ (the
Kolmogorov spectrum), while the exponent of the compressible part of the spectrum $q_{c}=2$ (for the Burgers turbulence with shock waves). The turbulent correlation time in $\boldsymbol{k}$ space is

$$
\tau(k)=\frac{2 \tau_{0}}{1+\sigma_{c}}\left[\bar{\tau}(k)+\sigma_{c} \bar{\tau}_{c}(k)\right]
$$

Note that for fully developed Kolmogorov like turbulence, $\sigma_{c}<1$ [54].

Integration in $\boldsymbol{k}$-space in Eq. (17) yields the turbulent heat flux $\langle\theta \boldsymbol{u}\rangle=\bar{T} \boldsymbol{V}^{\text {eff }}-D_{T} \nabla \bar{T}$, where the turbulent diffusivity $D_{T}$ and the effective pumping velocity $\boldsymbol{V}^{\mathrm{eff}}$ of the mean temperature field for large Péclet numbers are given by

$$
D_{T}=\frac{\tau_{0}\left\langle\boldsymbol{u}^{2}\right\rangle}{3}\left\{1+\frac{\gamma-1}{1+\sigma_{c}}\left[1-\frac{\sigma_{c}}{2\left(1+\sigma_{c}\right)}\left(\tilde{C}_{\sigma} q+\sigma_{c}\left(q_{c}-1\right)\right)\right]\right\}
$$




$$
\boldsymbol{V}^{\mathrm{eff}}=(\gamma-1) \frac{\tau_{0}\left\langle\boldsymbol{u}^{2}\right\rangle}{3\left(1+\sigma_{c}\right)}\left\{\frac{\sigma_{c}}{2}\left[1+\frac{\tilde{C}_{\sigma}}{2\left(1+\sigma_{c}\right)}\right] \boldsymbol{\lambda}_{u}+\left[1-\frac{\tilde{C}_{\sigma} \sigma_{c}}{2\left(1+\sigma_{c}\right)}\right] \boldsymbol{\lambda}_{P}\right\},
$$

and

$$
\tilde{C}_{\sigma}=\frac{2\left(q_{c}-1\right)}{q+q_{c}-2} .
$$

Equation (21) implies that for large Péclet numbers, compressibility effects decrease the turbulent diffusivity. Indeed, the derivative $\partial D_{T} / \partial \sigma_{c}$ is always negative when $\sigma_{c}\left(\tilde{C}_{\sigma} q-2 q_{c}\right)<\tilde{C}_{\sigma} q+2$. Since $\tilde{C}_{\sigma}>0$ and $\tilde{C}_{\sigma} q-2 q_{c}<0$ [the latter inequality is reduced to $\left(q_{c}-1\right)^{2}+(q-1)>0$ ], the derivative $\partial D_{T} / \partial \sigma_{c}$ is negative, i.e., compressibility effects do decrease the turbulent diffusivity.

For irrotational flow $\left(\sigma_{c} \gg 1\right)$, the turbulent diffusivity and the effective pumping velocity for large Péclet numbers are given by

$$
\begin{aligned}
D_{T} & =\frac{1}{3} \tau_{0}\left\langle\boldsymbol{u}^{2}\right\rangle\left[1-\frac{1}{2}(\gamma-1)\left(q_{c}-1\right)\right], \\
\boldsymbol{V}^{\mathrm{eff}} & =\left(\frac{\gamma-1}{6}\right) \tau_{0} \boldsymbol{\nabla}\left\langle\boldsymbol{u}^{2}\right\rangle .
\end{aligned}
$$

Equations (22) and (25) determine effective pumping velocity $\boldsymbol{V}^{\text {eff }}$ of the mean temperature field caused by the inhomogeneity of compressible turbulence and the gradient of the fluid pressure. Let us discuss mechanisms of the turbulent pumping effects. The first term $\left(\propto \sigma_{c} \boldsymbol{\nabla}\left\langle\boldsymbol{u}^{2}\right\rangle\right)$ in Eq. (22) implies that there is an additional contribution to the turbulent heat flux caused by the combined effect of the inhomogeneity of turbulence and compressibility of fluid flow. This effect results in increase of the mean temperature in the region with more intense velocity fluctuations in a compressible turbulence. This effect can be understood using the budget equation for the mean internal energy density $\bar{E}=c_{\mathrm{v}} \bar{T}$, where $c_{\mathrm{v}}$ is the specific heat at constant volume. In particular, one of the sources in the budget equation for the mean internal energy density is $-\langle p \boldsymbol{\nabla} \cdot \boldsymbol{u}\rangle[34]$, so that $\partial(\bar{\rho} \bar{E}) / \partial t \sim-\langle p \boldsymbol{\nabla} \cdot \boldsymbol{u}\rangle$, where $p$ are pressure fluctuations. As follows from the Bernoulli law, variations of the sum $\delta\left(p+\rho \boldsymbol{u}^{2} / 2\right) \approx 0$, so that $\delta p \approx-\delta\left(\rho \boldsymbol{u}^{2} / 2\right)$. This implies that the mean internal energy (and the mean temperature) is larger in the region with more intense compressible velocity fluctuations. The turbulent pumping effect of the mean temperature field caused by the joint effect of compressibility and inhomogeneity of turbulence is similar to a phenomenon of compressible turbophoresis for non-inertial particles or gaseous admixtures [7]. In particular, the expression for the effective pumping velocity for particles due to the compressible turbophoresis is proportional to $\boldsymbol{V}_{\text {particles }}^{\text {eff }} \propto \sigma_{c} \tau_{0} \boldsymbol{\nabla}\left\langle\boldsymbol{u}^{2}\right\rangle$.

The second term $(\propto \nabla \bar{P})$ in Eq. (22) determines an additional contribution to the turbulent heat flux caused by the gradient of the mean fluid pressure. This turbulent pumping increases the mean temperature in the regions with higher mean fluid temperature. The mechanism of this effect is the following. Since there is an outflow of fluid from the turbulent regions with higher mean fluid pressure, the fluid density decreases in these regions and temperature increases. This effect is similar to turbulent barodiffusion [11] of particles or gaseous admixtures.

Note that expressions (10) and (21) for turbulent diffusion coefficient of the mean temperature field in a compressible turbulence are different from those for the mean particle number density [5, 7]. Indeed, Eq. (10) for the temperature field contains an additional term, $(\gamma-2) T$ div $\boldsymbol{U}$, in comparison with Eq. (2) for the particle number density. Even for $\gamma=2$ when this additional term vanishes and the equations for the temperature field and the particle number density are similar, the expressions for turbulent diffusion coefficient for the mean temperature field in a compressible turbulence are different from those for the mean particle number density.

The main reason for this difference is as follows. Particles in a fluid flow is a two-phase system, while turbulent transport of fluid temperature is a one-phase system. Equation (4) for temperature fluctuations $\theta(\boldsymbol{x}, t)=T-\bar{T}$ has two source terms $I=-(\boldsymbol{u} \cdot \nabla) \bar{T}-(\gamma-1) \bar{T} \boldsymbol{\nabla} \cdot \boldsymbol{u}$, where the first term $-(\boldsymbol{u} \cdot \boldsymbol{\nabla}) \bar{T}$ contributes to turbulent diffusion $D_{T}$, while the second term $-(\gamma-1) \bar{T} \boldsymbol{\nabla} \cdot \boldsymbol{u}$ contributes to the effective pumping velocity $\boldsymbol{V}^{\text {eff }}$ of the mean temperature, so that the turbulent heat flux in a compressible turbulence is $\langle\theta \boldsymbol{u}\rangle=\bar{T} \boldsymbol{V}^{\mathrm{eff}}-D_{T} \boldsymbol{\nabla} \bar{T}$. The contribution $\left(\boldsymbol{V}^{\mathrm{eff}}\right) \nabla_{\bar{T}}=-D_{T}^{*} \nabla \bar{T} / \bar{T}$ to the effective pumping velocity $\boldsymbol{V}^{\text {eff }}{ }^{T}$ of the mean temperature due to the mean temperature gradient $\nabla \bar{T}$ is actually an additional contribution to the turbulent diffusivity $D_{T}$. Indeed, we can rewrite this contribution as

$$
\bar{T}\left(\boldsymbol{V}^{\mathrm{eff}}\right)_{\nabla_{\bar{T}}}=\bar{T}\left(-D_{T}^{*} \frac{\boldsymbol{\nabla} \bar{T}}{\bar{T}}\right)=-D_{T}^{*} \nabla \bar{T},
$$

where

$$
D_{T}^{*}=(\gamma-1) \frac{(q-1)}{3(q+1)} \frac{\tau_{0}\left\langle\boldsymbol{u}^{2}\right\rangle}{\left(1+\sigma_{c}\right)} \mathrm{Pe}
$$

for $\mathrm{Pe} \ll 1$, and

$$
D_{T}^{*}=(\gamma-1) \frac{\tau_{0}\left\langle\boldsymbol{u}^{2}\right\rangle}{3\left(1+\sigma_{c}\right)}\left(1-\frac{\tilde{C}_{\sigma} \sigma_{c}}{2\left(1+\sigma_{c}\right)}\right),
$$

for $\mathrm{Pe} \gg 1$. This is the main reason why the expressions for turbulent diffusion coefficient for the mean temperature field in a compressible turbulence are different from those for the mean particle number density.

Integration in $\boldsymbol{k}$-space in Eq. (18) yields the level of temperature fluctuations for large Péclet numbers 


$$
\left\langle\theta^{2}\right\rangle=8 f_{c}(\gamma-1)^{2}\left(\frac{\sigma_{c}}{1+\sigma_{c}}\right)^{3} \bar{T}^{2}+\frac{1}{9} \ell_{0}^{2}\left\{8(\nabla \bar{T})^{2}+(\gamma-1)\left[(\gamma+3)\left(\boldsymbol{\lambda}_{u} \cdot \boldsymbol{\nabla}\right)+2(5-\gamma)(\boldsymbol{\lambda} \cdot \boldsymbol{\nabla})\right] \bar{T}^{2}\right\},
$$

where the function $f_{c}\left(q, q_{c}, \sigma_{c}\right)$ depends on the degree of compressibility and the exponents of spectra for the incompressible and compressible parts of velocity fluctuations:

$$
f_{c}=\frac{q_{c}-1}{3 q_{c}-5}+\frac{2\left(q_{c}-1\right)}{\sigma_{c}\left(q+2 q_{c}-5\right)}+\frac{q_{c}-1}{\sigma_{c}^{2}\left(2 q+q_{c}-5\right)} .
$$

The first term in the right hand side of Eq. (29) determines a dominant contribution of the compressible part of velocity fluctuations to the level of temperature fluctuations. Here we neglect much smaller contributions $\sim \mathrm{O}\left[\ell_{0}^{2} /\left(L_{T} L_{u}\right)\right], \mathrm{O}\left[\ell_{0}^{2} /\left(L_{T} H_{\rho}\right)\right], \mathrm{O}\left[\ell_{0}^{2} / L_{T}^{2}\right]$, caused by the compressible part of velocity fluctuations. For small $\sigma_{c}$, the level of temperature fluctuations is determined by the other terms in Eq. (29) which are caused by the mean temperature gradient and the density stratified and inhomogeneous part of velocity fluctuations.

\section{TURBULENT COOLING}

In this section we discuss how a homogeneous compressible turbulence can cause a turbulent cooling of the surrounding fluid. Equation (3) for the mean temperature field $\bar{T}$ contains an additional sink term $I_{\mathrm{S}}=$ $-(\gamma-2)\langle\theta(\boldsymbol{\nabla} \cdot \boldsymbol{u})\rangle$ which can result in the turbulent cooling of the surrounding fluid for $\gamma<2$. Indeed, substituting Eq. (9) for the turbulent heat flux into Eq. (31), we obtain the equation for the mean temperature field $\bar{T}$ as

$$
\begin{aligned}
\frac{\partial \bar{T}}{\partial t} & +\boldsymbol{\nabla} \cdot\left[\bar{T} \boldsymbol{V}^{\mathrm{eff}}-\left(D+D_{T}\right) \boldsymbol{\nabla} \bar{T}\right]=\bar{J}_{\nu} \\
& -(\gamma-2)\langle\theta(\boldsymbol{\nabla} \cdot \boldsymbol{u})\rangle,
\end{aligned}
$$

where the sink term $I_{\mathrm{S}}$ in Eq. (31) for small Péclet numbers is given by

$$
I_{\mathrm{S}}=-(\gamma-1)(2-\gamma)\left(\frac{\sigma_{c}}{1+\sigma_{c}}\right) \operatorname{Pe} \frac{\bar{T}}{\tau_{0}},
$$

while for large Péclet numbers it is

$I_{\mathrm{S}}=-6(\gamma-1)(2-\gamma) \frac{\sigma_{c}}{\left(1+\sigma_{c}\right)^{2}} \frac{\bar{T}}{\tau_{0}}\left[\operatorname{Re}^{1 / 4}+\frac{\sigma_{c}}{4} \ln \operatorname{Re}\right]$.

In Eq. (33) for simplicity we determine $I_{\mathrm{S}}$ when that the exponent of the incompressible part of the turbulent kinetic energy spectrum for large Reynolds numbers is $q=5 / 3$, while the exponent of the compressible part of the spectrum is $q_{c}=2[55,[56]$.

Let us consider a simple case with a uniform mean temperature field. The heating source $\bar{J}_{\nu}$ in Eq. (31) caused by the viscous dissipation of the turbulent kinetic energy is given by

$$
\bar{J}_{\nu}=\frac{\nu}{c_{\mathrm{v}}}\left[\left\langle(\boldsymbol{\nabla} \times \boldsymbol{u})^{2}\right\rangle+\frac{4}{3}\left\langle(\boldsymbol{\nabla} \cdot \boldsymbol{u})^{2}\right\rangle\right],
$$

where $c_{\mathrm{v}}$ is the specific heat at constant volume. Here we use the equation for the turbulent kinetic energy density $E_{K}=\left\langle\rho \boldsymbol{u}^{2}\right\rangle / 2$ for compressible turbulence written as

$$
\frac{\partial E_{K}}{\partial t}+\operatorname{div} \boldsymbol{\Phi}_{K}=-\varepsilon_{K}+\Pi_{K},
$$

where

$$
\begin{aligned}
\boldsymbol{\Phi}_{K}= & -\nu\left[\langle\rho \boldsymbol{u} \times(\nabla \times \boldsymbol{u})\rangle+\frac{4}{3}\langle\rho \boldsymbol{u}(\boldsymbol{\nabla} \cdot \boldsymbol{u})\rangle\right] \\
& +\left\langle\boldsymbol{u}\left(\rho \boldsymbol{u}^{2} / 2\right)\right\rangle+\langle\boldsymbol{u} p\rangle
\end{aligned}
$$

is the flux of the density of turbulent kinetic energy, $p$ are fluid pressure fluctuations,

$$
\varepsilon_{K}=\nu\left[\left\langle\rho(\nabla \times \boldsymbol{u})^{2}\right\rangle+\frac{4}{3}\left\langle\rho(\boldsymbol{\nabla} \cdot \boldsymbol{u})^{2}\right\rangle\right]
$$

is the dissipation rate of the density of turbulent kinetic energy, and $\Pi_{K}=\langle\rho \boldsymbol{u} \cdot \boldsymbol{f}\rangle+\langle p(\boldsymbol{\nabla} \cdot \boldsymbol{u})\rangle$ is the production rate of the density of turbulent kinetic energy caused by the external force (e.g., by an external large-scale shear). The production term includes also the pressure-dilatation term $\langle p(\boldsymbol{\nabla} \cdot \boldsymbol{u})\rangle$ (see, e.g., [57, [58]). In the limit of low Mach numbers, the pressure-dilatation term in $\Pi_{K}$ is known to be much smaller than $\langle\rho \boldsymbol{u} \cdot \boldsymbol{f}\rangle$, and hence it can be safely neglected.

Using Eq. (19) for the second moment of velocity fluctuations in the background turbulence, we obtain that the viscous heating source $\bar{J}_{\nu}$ is given by

$$
\bar{J}_{\nu}=\frac{\left\langle\boldsymbol{u}^{2}\right\rangle}{2 \tau_{0}}\left(1+\sigma_{c}\right)^{-1}\left[1+\frac{8}{3} \sigma_{c} \operatorname{Re}^{-1 / 4}\right] .
$$

Turbulence can generate acoustic waves, and the rate of the energy radiated by the acoustic waves per unit mass for small Mach numbers is [59, 60]

$$
E_{\mathrm{w}}=\alpha \frac{\left\langle\boldsymbol{u}^{2}\right\rangle}{\tau_{0}} \mathrm{Ma}^{5},
$$

where $\alpha \sim 10-10^{2}$ is numerical coefficient, $\mathrm{Ma}=u_{\mathrm{rms}} / c_{\mathrm{s}}$ is the Mach number, $u_{\mathrm{rms}}=\left\langle\boldsymbol{u}^{2}\right\rangle^{1 / 2}$ and $c_{\mathrm{s}}=(\gamma \bar{P} / \bar{\rho})^{1 / 2}$ is the sound speed. The second term in Eq. (38) describes compressibility contribution to the rate of the viscous heating,

$$
\bar{J}_{\nu}^{(\mathrm{c})}=\frac{4}{3} \frac{\left\langle\boldsymbol{u}^{2}\right\rangle}{\tau_{0}} \frac{\sigma_{c}}{1+\sigma_{c}} \mathrm{Re}^{-1 / 4} .
$$


Assuming that the compressibility contribution to the viscous heating of turbulence $\bar{J}_{\nu}^{(\mathrm{c})}$ is compensated by the radiative wave energy density $E_{\mathrm{w}}$, we obtain that the degree of compressibility for small Mach numbers is given by

$$
\sigma_{c}=\frac{3 \alpha}{4} \mathrm{Ma}^{5} \operatorname{Re}^{1 / 4} .
$$

In the equilibrium, the total viscous heating $\bar{J}_{\nu}$ is compensated by the compressible cooling $I_{\mathrm{S}}$, so that the increase of the internal thermal energy caused by the viscous heating is given by

$$
c_{\mathrm{v}} \bar{T}_{c}=\frac{2\left\langle\boldsymbol{u}^{2}\right\rangle}{9 \alpha \mathrm{Ma}^{5} \operatorname{Re}^{1 / 2}} .
$$

Taking into account that the sound speed $c_{\mathrm{s}}$ depends on the mean temperature, we obtain from Eq. (42) that the increase of the internal thermal energy caused by the viscous heating is given by

$$
c_{\mathrm{v}} \bar{T}_{c}=C_{*}\left\langle\boldsymbol{u}^{2}\right\rangle \operatorname{Re}^{1 / 3},
$$

where $C_{*}=(9 \alpha / 2)^{2 / 3} /[\gamma(\gamma-1)]^{5 / 3}$. Equation (43) can be rewritten in terms of the Mach number $\mathrm{Ma}=u_{\mathrm{rms}} / c_{\mathrm{s}}$ as

$$
\mathrm{Ma}=\left[\frac{2 \gamma(\gamma-1)}{9 \alpha}\right]^{1 / 3} \operatorname{Re}^{-1 / 6} .
$$

For example, taking parameters typical for the atmospheric turbulence, $\ell_{0}=10^{2} \mathrm{~cm}$ and $u_{\mathrm{rms}}=2.7 \times 10^{2}$ $\mathrm{cm} / \mathrm{s}$ and $\alpha=10$, we obtain that $T_{c}=286 \mathrm{~K}$.

\section{DISCUSSION AND CONCLUSIONS}

In the present study we have investigated compressibility effects on turbulent transport of the mean temperature field. We use the quasi-linear approach for study turbulent transport for small Péclet numbers. When nonlinear effects are much stronger than the molecular diffusion (i.e., for large Péclet numbers), we apply the spectral $\tau$ approach. Similarly to turbulent transport of particles and magnetic fields, the compressibility decreases the turbulent diffusivity of the mean temperature field, but the expression for turbulent diffusivity for the mean temperature field in a compressible turbulence is different from those for turbulent diffusivity of the mean particle number density and turbulent magnetic diffusivity of the mean magnetic field.

We have found also turbulent pumping of the mean temperature field due to joint effects of the fluid flow compressibility and inhomogeneity of turbulence. This effect causes an increase of the mean temperature in the regions of more intense velocity fluctuations. Similar compressibility effect referred to compressible turbophoresis [7], results in a pumping of non-inertial particles or gaseous admixtures from regions of low to high turbulent intensity. Turbulent pumping also can be due to the gradients of the mean fluid pressure resulting in increase of the mean temperature in the regions with increased mean fluid pressure, similarly to phenomenon of turbulent barodiffusion of particles and gaseous admixtures.

Due to compressibility, there is an additional sink term in the equation for the mean fluid temperature, causing a turbulent cooling in homogeneous turbulence. This implies that there can be an equilibrium in a compressible homogeneous turbulence with a uniform mean fluid temperature, where the heating caused by the viscous dissipation of the turbulent kinetic energy can be compensated by the turbulent cooling caused by the fluid compressibility. Such effect does not exist in turbulent transport of particles or gaseous admixtures in a compressible fluid flow.

To derive expressions for the turbulent heat flux and the level of temperature fluctuations for large Péclet and Reynolds numbers in a compressible inhomogeneous and density stratified turbulence, we apply the spectral $\tau$ approach (see Sect. IV). The $\tau$ approach reproduces many well-known phenomena found by other methods in turbulent transport of particles, temperature and magnetic fields, in turbulent convection and stably stratified turbulent flows (see for a review, [5]). In turbulent transport, the $\tau$ approach yields correct formulae for turbulent diffusion, turbulent thermal diffusion and turbulent barodiffusion 9 11, 61. The phenomenon of turbulent thermal diffusion was predicted using the stochastic calculus (the path integral approach). This effect was also reproduced using the quasi-linear approach, the spectral $\tau$ approach and the renormalization approach.

The $\tau$ approach reproduces the well-known $k^{-7 / 3}$ spectrum of anisotropic velocity fluctuations in a sheared turbulence (see 62]). This spectrum was previously found in analytical, numerical, laboratory studies and was observed in the atmospheric turbulence (see, e.g., [63]). In the turbulent boundary layer problems, the $\tau$ approach yields correct expressions for turbulent viscosity, turbulent thermal conductivity and the turbulent heat flux [2, 39]. This approach also describes the counter wind turbulent heat flux and the Deardorff's heat flux in convective boundary layers (see [62]). These phenomena were studied using different approaches (see, e.g., [2, 39, 64]).

In magnetohydrodynamics, the $\tau$ approach reproduces many well-known phenomena found by different methods, e.g., the $\tau$ approximation yields correct formulae for the $\alpha$-effect, the turbulent diamagnetic and paramagnetic velocities, the turbulent magnetic diffusion, the $\boldsymbol{\Omega} \times \mathbf{J}$ effect and the $\kappa$-effect [8, 65, 66] .

\section{ACKNOWLEDGMENTS}

We have benefited from stimulating discussions with Axel Brandenburg and Michael Liberman. This research was supported in part by Ministry of Science and Technology (grant No. 3-16516) and PAZY Foundation of 
the Israel Atomic Energy Commission (IAEC) (grant No. functions are written as follows:

122-2020).

\section{Appendix A: Multi-scale approach}

In the framework of the multi-scale approach [42], the non-instantaneous two-point second-order correlation

$$
\begin{aligned}
\left\langle\theta\left(\boldsymbol{x}, t_{1}\right) u_{j}\left(\boldsymbol{y}, t_{2}\right)\right\rangle & =\int\left\langle\theta\left(\boldsymbol{k}_{1}, \omega_{1}\right) u_{j}\left(\boldsymbol{k}_{2}, \omega_{2}\right)\right\rangle \exp \left[i\left(\boldsymbol{k}_{1} \cdot \boldsymbol{x}+\boldsymbol{k}_{2} \cdot \boldsymbol{y}\right)+i\left(\omega_{1} t_{1}+\omega_{2} t_{2}\right)\right] d \omega_{1} d \omega_{2} d \boldsymbol{k}_{1} d \boldsymbol{k}_{2} \\
& =\int F_{j}(\boldsymbol{k}, \omega, t, \boldsymbol{R}) \exp [i \boldsymbol{k} \cdot \boldsymbol{r}+i \omega \tilde{\tau}] d \omega d \boldsymbol{k} \\
\left\langle\theta\left(\boldsymbol{x}, t_{1}\right) \theta\left(\boldsymbol{y}, t_{2}\right)\right\rangle & =\int\left\langle\theta\left(\boldsymbol{k}_{1}, \omega_{1}\right) \theta\left(\boldsymbol{k}_{2}, \omega_{2}\right)\right\rangle \exp \left[i\left(\boldsymbol{k}_{1} \cdot \boldsymbol{x}+\boldsymbol{k}_{2} \cdot \boldsymbol{y}\right)+i\left(\omega_{1} t_{1}+\omega_{2} t_{2}\right)\right] d \omega_{1} d \omega_{2} d \boldsymbol{k}_{1} d \boldsymbol{k}_{2} \\
& =\int E_{\theta}(\boldsymbol{k}, \omega, t, \boldsymbol{R}) \exp [i \boldsymbol{k} \cdot \boldsymbol{r}+i \omega \tau \tilde{\tau}] d \omega d \boldsymbol{k}
\end{aligned}
$$

where

$$
\begin{aligned}
& F_{j}(\boldsymbol{k}, \omega, \boldsymbol{R}, t)=\int\left\langle\theta\left(\boldsymbol{k}_{1}, \omega_{1}\right) u_{j}\left(\boldsymbol{k}_{2}, \omega_{2}\right)\right\rangle \exp [i \Omega t \\
& \quad+i \boldsymbol{K} \cdot \boldsymbol{R}] d \Omega d \boldsymbol{K} . \\
& E_{\theta}(\boldsymbol{k}, \omega, \boldsymbol{R}, t)=\int\left\langle\theta\left(\boldsymbol{k}_{1}, \omega_{1}\right) \theta\left(\boldsymbol{k}_{2}, \omega_{2}\right)\right\rangle \exp [i \Omega t \\
& \quad+i \boldsymbol{K} \cdot \boldsymbol{R}] d \Omega d \boldsymbol{K} .
\end{aligned}
$$

Here we introduce large-scale variables: $\boldsymbol{R}=(\boldsymbol{x}+\boldsymbol{y}) / 2$, $\boldsymbol{K}=\boldsymbol{k}_{1}+\boldsymbol{k}_{2}, \quad t=\left(t_{1}+t_{2}\right) / 2, \Omega=\omega_{1}+\omega_{2}$, and smallscale variables: $\boldsymbol{r}=\boldsymbol{x}-\boldsymbol{y}, \boldsymbol{k}=\left(\boldsymbol{k}_{1}-\boldsymbol{k}_{2}\right) / 2, \tilde{\tau}=t_{1}-t_{2}$, $\omega=\left(\omega_{1}-\omega_{2}\right) / 2$. This implies that $\omega_{1}=\omega+\Omega / 2$, $\omega_{2}=-\omega+\Omega / 2, \boldsymbol{k}_{1}=\boldsymbol{k}+\boldsymbol{K} / 2$, and $\boldsymbol{k}_{2}=-\boldsymbol{k}+\boldsymbol{K} / 2$. Mean-fields depend on the large-scale variables, while fluctuations depend on the small-scale variables. Similarly to Eqs. (A1) A4 , correlation function for velocity fluctuations reads

$$
\begin{aligned}
& f_{i j}(\boldsymbol{k}, \omega, \boldsymbol{R}, t)=\int\left\langle u_{i}\left(\boldsymbol{k}_{1}, \omega_{1}\right) u_{j}\left(\boldsymbol{k}_{2}, \omega_{2}\right)\right\rangle \exp [i \Omega t \\
& \quad+i \boldsymbol{K} \cdot \boldsymbol{R}] d \Omega d \boldsymbol{K} .
\end{aligned}
$$

After separation into slow and fast variables and calculating the functions $F_{j}(\boldsymbol{k}, \omega, \boldsymbol{R}, t)$ and $E_{\theta}(\boldsymbol{k}, \omega, \boldsymbol{R}, t)$, Eqs. A1 and (A2) in the limit of $\boldsymbol{r} \rightarrow \mathbf{0}$ and $\tilde{\tau} \rightarrow 0$ allow us to determine the turbulent flux of the temperature field and the level of temperature fluctuations in physical space:

$$
\begin{aligned}
\left\langle\theta(\boldsymbol{x}, t) u_{j}(\boldsymbol{x}, t)\right\rangle & =\int F_{j}(\boldsymbol{k}, \omega, \boldsymbol{R}, t) d \omega d \boldsymbol{k}, \\
\langle\theta(\boldsymbol{x}, t) \theta(\boldsymbol{x}, t)\rangle & =\int E_{\theta}(\boldsymbol{k}, \omega, \boldsymbol{R}, t) d \omega d \boldsymbol{k} .
\end{aligned}
$$

\section{Appendix B: Derivation of Eqs. (5)-(7)}

We rewrite Eq. (4) in Fourier space and find solution of this equation as

$$
\begin{gathered}
\theta(\boldsymbol{k}, \omega)=-i\left[(\gamma-1) \int \bar{T}(\boldsymbol{Q})\left(k_{i}-Q_{i}\right) u_{i}(\boldsymbol{k}-\boldsymbol{Q}, \omega) d \boldsymbol{Q}\right. \\
\left.+\int Q_{i} \bar{T}(\boldsymbol{Q}) u_{i}(\boldsymbol{k}-\boldsymbol{Q}, \omega) d \boldsymbol{Q}\right] G_{D}(\boldsymbol{k}, \omega),
\end{gathered}
$$

where $G_{D}(\boldsymbol{k}, \omega)=\left(D \boldsymbol{k}^{2}+i \omega\right)^{-1}$. Using Eqs. A6 and (B1), we determine the functions $F_{j}(\boldsymbol{k}, \boldsymbol{R})$ and $E_{\theta}(\boldsymbol{k}, \boldsymbol{R})$ as

$$
\begin{aligned}
F_{j}(\boldsymbol{k}, \boldsymbol{R})= & -i \int\left[(\gamma-1)\left(k_{i}+\frac{K_{i}}{2}-Q_{i}\right)+Q_{i}\right] G_{D}(\boldsymbol{k}+\boldsymbol{K} / 2)\left\langle u_{i}(\boldsymbol{k}+\boldsymbol{K} / 2-\boldsymbol{Q}) u_{j}(-\boldsymbol{k}+\boldsymbol{K} / 2)\right\rangle \\
& \times \bar{T}(\boldsymbol{Q}) \exp (i \boldsymbol{K} \cdot \boldsymbol{R}) d \boldsymbol{K} d \boldsymbol{Q}, \\
E_{\theta}(\boldsymbol{k}, \boldsymbol{R})= & -\frac{i}{2} \int\left\{\left[(\gamma-1)\left(k_{i}+\frac{K_{i}}{2}-Q_{i}\right)+Q_{i}\right] G_{D}(\boldsymbol{k}+\boldsymbol{K} / 2)\left\langle\theta(-\boldsymbol{k}+\boldsymbol{K} / 2) u_{i}(\boldsymbol{k}+\boldsymbol{K} / 2-\boldsymbol{Q})\right\rangle\right. \\
& \left.+\left[(\gamma-1)\left(-k_{i}+\frac{K_{i}}{2}-Q_{i}\right)+Q_{i}\right] G_{D}(-\boldsymbol{k}+\boldsymbol{K} / 2)\left\langle\theta(\boldsymbol{k}+\boldsymbol{K} / 2) u_{i}(-\boldsymbol{k}+\boldsymbol{K} / 2-\boldsymbol{Q})\right\rangle\right\}
\end{aligned}
$$


where the functions $F_{j}, G_{D}$ and $u_{i}$ depend also on $\omega$, and $\bar{T}$ depend on $t$ as well. To simplify the notations, we do not show these dependencies here. To determine $f_{i j}(\boldsymbol{k}, \boldsymbol{K}, \boldsymbol{Q})=\left\langle u_{i}(\boldsymbol{k}+\boldsymbol{K} / 2-\boldsymbol{Q}) u_{j}(-\boldsymbol{k}+\boldsymbol{K} / 2)\right\rangle$, we use the following new variables:

$$
\begin{gathered}
\tilde{\boldsymbol{k}}=\left(\tilde{\boldsymbol{k}}_{1}-\tilde{\boldsymbol{k}}_{2}\right) / 2=\boldsymbol{k}-\boldsymbol{Q} / 2, \\
\tilde{\boldsymbol{K}}=\tilde{\boldsymbol{k}}_{1}+\tilde{\boldsymbol{k}}_{2}=\boldsymbol{K}-\boldsymbol{Q},
\end{gathered}
$$

where

$$
\tilde{\boldsymbol{k}}_{1}=\boldsymbol{k}+\boldsymbol{K} / 2-\boldsymbol{Q}, \quad \tilde{\boldsymbol{k}}_{2}=-\boldsymbol{k}+\boldsymbol{K} / 2 .
$$

Since $|\boldsymbol{Q}| \ll|\boldsymbol{k}|$ and $|\boldsymbol{K}| \ll|\boldsymbol{k}|$, we use the Taylor expansion

$$
\begin{aligned}
& f_{i j}(\boldsymbol{k}-\boldsymbol{Q} / 2, \boldsymbol{K}-\boldsymbol{Q})=f_{i j}(\boldsymbol{k}, \boldsymbol{K}-\boldsymbol{Q})-\frac{1}{2} \frac{\partial f_{i j}}{\partial k_{m}} Q_{m} \\
& \quad+O\left(\boldsymbol{Q}^{2}\right),
\end{aligned}
$$

$$
\begin{aligned}
& G_{D}(\boldsymbol{k}+\boldsymbol{K} / 2)=G_{D}(\boldsymbol{k})\left[1-D(\boldsymbol{k} \cdot \boldsymbol{K}) G_{D}(\boldsymbol{k})\right] \\
& \quad+O\left(\boldsymbol{K}^{2}\right) .
\end{aligned}
$$

In the similar way we calculate other terms in Eqs. (B2)(B3). Using Eqs. (B2) - (B8), we arrive at expressions (5) (6) for the turbulent heat flux and the level of temperature fluctuations in Fourier space for small Péclet numbers.

To derive Eq. (7), the second rank tensor $f_{i j}^{(0)}$ is constructed as a linear combination of symmetric tensors, $\delta_{i j}$ and $k_{i j}$, with respect to the indexes $i$ and $j$, and nonsymmetric tensors: $k_{i} \lambda_{j}, k_{j} \lambda_{i}, k_{i} \nabla_{j}\left\langle\boldsymbol{u}^{2}\right\rangle$ and $k_{j} \nabla_{i}\left\langle\boldsymbol{u}^{2}\right\rangle$. We consider here only linear effects in $\boldsymbol{\lambda}$ and $\boldsymbol{\nabla}\left\langle\boldsymbol{u}^{2}\right\rangle$. To determine unknown coefficients multiplying by these tensors, we use the following conditions in the derivation of Eq. (77): $\left\langle\boldsymbol{u}^{2}\right\rangle=\int f_{i i}^{(0)}(\boldsymbol{k}, \omega, \boldsymbol{K}) \exp (i \boldsymbol{K} \cdot \boldsymbol{R}) d \boldsymbol{k} d \omega d \boldsymbol{K}$, $f_{i j}^{(0)}(\boldsymbol{k}, \omega, \boldsymbol{K})=f_{j i}^{*(0)}(\boldsymbol{k}, \omega, \boldsymbol{K})=f_{j i}^{(0)}(-\boldsymbol{k}, \omega, \boldsymbol{K})$, and
The normalization conditions for the functions $\Phi(\omega)$, $E(k)$ and $E_{c}(k)$ in Eq. (77) are $\int_{-\infty}^{\infty} \Phi(\omega) d \omega=1$, $\int_{k_{0}}^{k_{d}} E(k) d k=1$ and $\int_{k_{0}}^{k_{d}} E_{c}(k) d k=1$. For very low Mach numbers, i.e., when the parameter $\sigma_{c}$ is very small, the continuity equation can be written in the anelastic approximation, $\operatorname{div}(\bar{\rho} \boldsymbol{u})=0$, which implies that $\left(i k_{i}+i K_{i} / 2-\lambda_{i}\right) f_{i j}^{(0)}(\boldsymbol{k}, \omega, \boldsymbol{K})=0$ and $\left(-i k_{j}+i K_{j} / 2-\right.$ $\left.\lambda_{j}\right) f_{i j}^{(0)}(\boldsymbol{k}, \omega, \boldsymbol{K})=0$.

For the integration over $\omega$ in Eqs. (5) and (6), we use the following identities:

$$
\begin{aligned}
& \int_{-\infty}^{\infty} \frac{d \omega}{\left( \pm i \omega+D k^{2}\right)\left(\omega^{2}+\tau_{0}^{-2}\right)}=\frac{\pi \tau_{0}}{\tau_{0}^{-1}+D k^{2}} \approx \frac{\pi \tau_{0}}{D k^{2}} \\
& \int_{-\infty}^{\infty} \frac{d \omega}{\left(i \omega+D k^{2}\right)\left(-i \omega+D k^{2}\right)\left(\omega^{2}+\tau_{0}^{-2}\right)} \\
& \quad=\frac{\pi \tau_{0}}{D k^{2}\left(\tau_{0}^{-1}+D k^{2}\right)} \approx \frac{\pi \tau_{0}}{\left(D k^{2}\right)^{2}}
\end{aligned}
$$

which are determined in the limit when the correlation time $\tau_{0} \gg\left(D^{(\theta)} k^{2}\right)^{-1}$. For the integration over angles in $\boldsymbol{k}$ space in Eqs. (5) and (6), we use the following identity:

$$
\int_{0}^{2 \pi} d \varphi \int_{0}^{\pi} \sin \vartheta d \vartheta \frac{k_{i} k_{j}}{k^{2}}=\frac{4 \pi}{3} \delta_{i j} .
$$

For the integration over $k$ in Eqs. (5) and (6), we use the following identities:

$$
\begin{aligned}
& \int_{k_{0}}^{k_{d}} \frac{E(k)}{k^{2}} d k=\frac{q-1}{q+1} \ell_{0}^{2}, \\
& \int_{k_{0}}^{k_{d}} \frac{E(k)}{k^{4}} d k=\frac{q-1}{q+3} \ell_{0}^{4} .
\end{aligned}
$$

\section{Appendix C: Derivation of Eqs. (14) and (15)}

In this Appendix we derive Eqs. (14) and (15) for large Péclet and Reynolds numbers. Using Eq. (4) for the temperature fluctuations $\theta$ and the Navier-Stokes equation for the velocity $\boldsymbol{u}$ written in Fourier space, we derive equations for the following correlation functions:

$$
\begin{aligned}
F_{j}(\boldsymbol{k}, \boldsymbol{R})= & \int\left\langle\theta(\boldsymbol{k}+\boldsymbol{K} / 2) u_{j}(-\boldsymbol{k}+\boldsymbol{K} / 2)\right\rangle \\
& \times \exp [i \boldsymbol{K} \cdot \boldsymbol{R}] d \boldsymbol{K}, \\
E_{\theta}(\boldsymbol{k}, \boldsymbol{R})= & \int\langle\theta(\boldsymbol{k}+\boldsymbol{K} / 2) \theta(-\boldsymbol{k}+\boldsymbol{K} / 2)\rangle \\
& \times \exp [i \boldsymbol{K} \cdot \boldsymbol{R}] d \boldsymbol{K} .
\end{aligned}
$$

For brevity of notations we omit the large-scale variable $t$ in the functions $F_{j}(\boldsymbol{k}, \boldsymbol{R}, t), E_{\theta}(\boldsymbol{k}, \boldsymbol{R}, t)$ and the mean temperature $\bar{T}(\boldsymbol{R}, t)$. 
To derive evolution equations in the Fourier space for the turbulent heat flux $F_{j}(\boldsymbol{k}, \boldsymbol{R})$ and the level of temperature fluctuations $E_{\theta}(\boldsymbol{k}, \boldsymbol{R})$, we rewrite Eq. (4) for the temperature fluctuations in $\boldsymbol{k}$ space as

$$
\begin{aligned}
& \frac{\partial \theta(\boldsymbol{k})}{\partial t}=-i\left[(\gamma-1) \int \bar{T}(\boldsymbol{Q})\left(k_{i}-Q_{i}\right) u_{i}(\boldsymbol{k}-\boldsymbol{Q}) d \boldsymbol{Q}\right. \\
& \left.+\int Q_{i} \bar{T}(\boldsymbol{Q}) u_{i}(\boldsymbol{k}-\boldsymbol{Q}) d \boldsymbol{Q}\right]-\mathcal{Q}(\boldsymbol{k}),
\end{aligned}
$$

where $\mathcal{Q}(\boldsymbol{k})$ are the nonlinear terms written in $\boldsymbol{k}$ space. For brevity of notations we omit below the variable $t$ in the functions $\bar{T}(\boldsymbol{Q}, t), \theta(\boldsymbol{k}, t), \theta^{(\mathrm{N})}(\boldsymbol{k}, t)$ and $u_{i}(\boldsymbol{k}, t)$.

Using Eq. (C3) for the temperature fluctuations $\theta$ written in Fourier space, we derive equations for the instantaneous two-point correlation functions $F_{j}(\boldsymbol{k}, \boldsymbol{R})$ and $E_{\theta}(\boldsymbol{k}, \boldsymbol{R})$ defined by Eqs. (C1) and (C2). To this end we use the identities:

$$
\begin{aligned}
& \frac{\partial}{\partial t}\left\langle\theta\left(\boldsymbol{k}_{1}, t\right) u_{j}\left(\boldsymbol{k}_{2}, t\right)\right\rangle=\left\langle\frac{\partial \theta\left(\boldsymbol{k}_{1}, t\right)}{\partial t} u_{j}\left(\boldsymbol{k}_{2}, t\right)\right\rangle \\
& \quad+\left\langle\theta\left(\boldsymbol{k}_{1}, t\right) \frac{\partial u_{j}\left(\boldsymbol{k}_{2}, t\right)}{\partial t}\right\rangle
\end{aligned}
$$

$$
\begin{aligned}
& \frac{\partial}{\partial t}\left\langle\theta\left(\boldsymbol{k}_{1}, t\right) \theta\left(\boldsymbol{k}_{2}, t\right)\right\rangle=\left\langle\frac{\partial \theta\left(\boldsymbol{k}_{1}, t\right)}{\partial t} \theta\left(\boldsymbol{k}_{2}, t\right)\right\rangle \\
& \quad+\left\langle\theta\left(\boldsymbol{k}_{1}, t\right) \frac{\partial \theta\left(\boldsymbol{k}_{2}, t\right)}{\partial t}\right\rangle .
\end{aligned}
$$

Equations (C3)-C5 yield the dynamic equations as

$$
\begin{aligned}
& \frac{\partial F_{j}(\boldsymbol{k}, \boldsymbol{R})}{\partial t}=J_{j}(\boldsymbol{k}, \boldsymbol{R})+\hat{\mathcal{M}} F_{j}^{(\mathrm{III})}(\boldsymbol{k}, \boldsymbol{R}), \\
& \frac{\partial E_{\theta}(\boldsymbol{k}, \boldsymbol{R})}{\partial t}=S(\boldsymbol{k}, \boldsymbol{R})+\hat{\mathcal{M}} E_{\theta}^{(\mathrm{III})}(\boldsymbol{k}, \boldsymbol{R}),
\end{aligned}
$$

where

$$
\begin{gathered}
\hat{\mathcal{M}} F_{j}^{(\mathrm{III})}(\boldsymbol{k}, \boldsymbol{R})=\int\left[\left\langle\theta\left(\boldsymbol{k}_{1}\right) \frac{\partial u_{j}\left(\boldsymbol{k}_{2}\right)}{\partial t}\right\rangle\right. \\
\left.-\left\langle\mathcal{Q}\left(\boldsymbol{k}_{1}\right) u_{j}\left(\boldsymbol{k}_{2}\right)\right\rangle\right] \exp [i \boldsymbol{K} \cdot \boldsymbol{R}] d \boldsymbol{K} \\
\hat{\mathcal{M}} E_{\theta}^{(\mathrm{III})}(\boldsymbol{k}, \boldsymbol{R})=-\int\left[\left\langle\theta\left(\boldsymbol{k}_{1}\right) \mathcal{Q}\left(\boldsymbol{k}_{2}\right)\right\rangle\right. \\
\left.-\left\langle\mathcal{Q}\left(\boldsymbol{k}_{1}\right) \theta\left(\boldsymbol{k}_{2}\right)\right\rangle\right] \exp [i \boldsymbol{K} \cdot \boldsymbol{R}] d \boldsymbol{K}
\end{gathered}
$$

are the third-order moment terms in $\boldsymbol{k}$ space appearing due to the nonlinear terms, and

$$
\begin{aligned}
J_{j}(\boldsymbol{k}, \boldsymbol{R})= & -i \int\left[(\gamma-1)\left(\boldsymbol{k}_{i}+K_{i} / 2-Q_{i}\right)+Q_{i}\right]\left\langle u_{i}(\boldsymbol{k}+\boldsymbol{K} / 2-\boldsymbol{Q}) u_{j}(-\boldsymbol{k}+\boldsymbol{K} / 2)\right\rangle \bar{T}(\boldsymbol{Q}) \exp (i \boldsymbol{K} \cdot \boldsymbol{R}) d \boldsymbol{K} d \boldsymbol{Q}, \\
S(\boldsymbol{k}, \boldsymbol{R})= & -i \int\left\{\left[(\gamma-1)\left(\boldsymbol{k}_{j}+K_{j} / 2-Q_{j}\right)+Q_{j}\right]\left\langle\theta(-\boldsymbol{k}+\boldsymbol{K} / 2) u_{j}(\boldsymbol{k}+\boldsymbol{K} / 2-\boldsymbol{Q})\right\rangle\right. \\
& \left.+\left[(\gamma-1)\left(-\boldsymbol{k}_{j}+K_{j} / 2-Q_{j}\right)+Q_{j}\right]\left\langle\theta(\boldsymbol{k}+\boldsymbol{K} / 2) u_{j}(-\boldsymbol{k}+\boldsymbol{K} / 2-\boldsymbol{Q})\right\rangle\right\} \bar{T}(\boldsymbol{Q}) \exp (i \boldsymbol{K} \cdot \boldsymbol{R}) d \boldsymbol{K} d \boldsymbol{Q} .
\end{aligned}
$$

To derive Eq. (14), we perform calculations in Eq. C10 which are similar to those in Eqs. (B4)-B7). To determine $\left\langle\theta\left(\tilde{\boldsymbol{k}}_{1}\right) u_{j}\left(\tilde{\boldsymbol{k}}_{2}\right)\right\rangle$ in Eq. (C11), we use new variables:

$$
\begin{aligned}
\tilde{\boldsymbol{k}} & =\left(\tilde{\boldsymbol{k}}_{1}-\tilde{\boldsymbol{k}}_{2}\right) / 2=-\boldsymbol{k}+\boldsymbol{Q} / 2, \\
\tilde{\boldsymbol{K}} & =\tilde{\boldsymbol{k}}_{1}+\tilde{\boldsymbol{k}}_{2}=\boldsymbol{K}-\boldsymbol{Q},
\end{aligned}
$$

where

$$
\tilde{\boldsymbol{k}}_{1}=-\boldsymbol{k}+\boldsymbol{K} / 2, \quad \tilde{\boldsymbol{k}}_{2}=\boldsymbol{k}+\boldsymbol{K} / 2-\boldsymbol{Q} .
$$

Since $|\boldsymbol{Q}| \ll|\boldsymbol{k}|$ and $|\boldsymbol{K}| \ll|\boldsymbol{k}|$, we use the Taylor expansion

$$
\begin{gathered}
\left\langle\theta\left(\tilde{\boldsymbol{k}}_{1}\right) u_{j}\left(\tilde{\boldsymbol{k}}_{2}\right)\right\rangle=F_{j}(\tilde{\boldsymbol{k}}, \tilde{\boldsymbol{K}})=F_{j}(-\boldsymbol{k}, \tilde{\boldsymbol{K}})+\frac{Q_{m}}{2} \frac{\partial F_{j}}{\partial \tilde{k}_{m}} \\
+O\left(\boldsymbol{Q}^{2}\right)=\left(1-\frac{Q_{m}}{2} \frac{\partial}{\partial k_{m}}\right) F_{j}(-\boldsymbol{k}, \tilde{\boldsymbol{K}})+O\left(\boldsymbol{Q}^{2}\right)
\end{gathered}
$$

Similarly,

$$
\left\langle\theta\left(\tilde{\boldsymbol{k}}_{3}\right) u_{j}\left(\tilde{\boldsymbol{k}}_{4}\right)\right\rangle=\left(1+\frac{Q_{m}}{2} \frac{\partial}{\partial k_{m}}\right) F_{j}(\boldsymbol{k}, \tilde{\boldsymbol{K}})+O\left(\boldsymbol{Q}^{2}\right),
$$

where

$$
\tilde{\boldsymbol{k}}_{3}=\boldsymbol{k}+\boldsymbol{K} / 2, \quad \tilde{\boldsymbol{k}}_{4}=-\boldsymbol{k}+\boldsymbol{K} / 2-\boldsymbol{Q} .
$$

Substituting Eqs. (C15 and (C16) into Eq. (C11), neglecting the terms $O\left(\boldsymbol{Q}^{2} ; \boldsymbol{K}^{2}\right)$, and returning to the physical space in the large-scale variables, we obtain Eqs. (14) and (15).

To determine the turbulent heat flux and the level of temperature fluctuations, we use the following identities for integration over $k$ in Eqs. (17) and (18):

$$
\int_{k_{0}}^{k_{\nu}} \tau(k)\left[E(k)+\sigma_{c} E_{c}(k)\right] d k=\tau_{0}\left(1+\sigma_{c}\right),
$$




$$
\begin{gathered}
\int_{k_{0}}^{k_{\nu}} \tau(k) E(k) d k=\tau_{0}\left[1-\frac{\tilde{C}_{\sigma} \sigma_{c}}{2\left(1+\sigma_{c}\right)}\right], \\
\int_{k_{0}}^{k_{\nu}} \tau(k) E_{c}(k) d k=\tau_{0}\left[1+\frac{\tilde{C}_{\sigma}}{2\left(1+\sigma_{c}\right)}\right], \\
\int_{k_{0}}^{k_{\nu}} \tau(k) k^{2} E_{c}(k) d k=\frac{6 \tau_{0}}{\ell_{0}^{2}}\left(1+\sigma_{c}\right)^{-1}\left[\operatorname{Re}^{1 / 4}\right. \\
\left.+\frac{\sigma_{c}}{4} \ln \operatorname{Re}\right] \\
\int_{k_{0}}^{k_{\nu}} \frac{d \tau(k)}{d k} E_{c}(k) k d k=-\frac{\tau_{0}\left(q_{c}-1\right) \sigma_{c}}{1+\sigma_{c}} \\
\times\left[1+\frac{2(q-1)}{\sigma_{c}\left(q+q_{c}-2\right)}\right], \\
\int_{k_{0}}^{k_{\nu}} \tau^{2}(k) k^{2} E_{c}(k) d k= \\
\times f_{c}\left(\frac{\tau_{0}}{\ell_{0}}\right)^{2}\left(\frac{\sigma_{c}}{1+\sigma_{c}}\right)^{2},
\end{gathered}
$$

$$
\int_{k_{0}}^{k_{\nu}} \tau^{2}(k)\left[E(k)+\sigma_{c} E_{c}(k)\right] d k=\frac{4}{3} \tau_{0}^{2}\left(1+\sigma_{c}\right),
$$$$
\int_{k_{0}}^{k_{\nu}} \tau^{2}(k) E_{c}(k) d k=\frac{4}{3} \tau_{0}^{2} f_{*}\left(\frac{\sigma_{c}}{1+\sigma_{c}}\right)^{2}
$$$$
\int_{k_{0}}^{k_{\nu}} \tau^{2}(k) E(k) d k=\frac{4}{3} \tau_{0}^{2}\left(1+\sigma_{c}\right)\left[1-f_{*}\left(\frac{\sigma_{c}}{1+\sigma_{c}}\right)^{3}\right]
$$

where

$$
f_{*}=1+\frac{6\left(q_{c}-1\right)}{\sigma_{c}\left(q+2 q_{c}-3\right)}+\frac{3\left(q_{c}-1\right)}{\sigma_{c}^{2}\left(2 q+q_{c}-3\right)} .
$$

[1] A. S. Monin and A. M. Yaglom, Statistical Fluid Mechanics (MIT Press, Cambridge, Massachusetts, 1971), v. 1.

[2] A. S. Monin and A. M. Yaglom, Statistical Fluid Mechanics (MIT Press, Cambridge, Massachusetts, 1975), v. 2.

[3] Ya. B. Zeldovich, A. A. Ruzmaikin, and D. D. Sokoloff, The Almighty Chance (Word Scientific Publ., London, 1990).

[4] L. I. Zaichik, V. M. Alipchenkov, and E. G. Sinaiski, Particles in Turbulent Flows (John Wiley \& Sons, NY, 2008).

[5] I. Rogachevskii, Introduction to Turbulent Transport of Particles, Temperature and Magnetic Fields (Cambridge University Press, Cambridge, 2021).

[6] K.-H. Rädler, A. Brandenburg, F. Del Sordo and M. Rheinhardt, Phys. Rev. E 84, 046321 (2011).

[7] I. Rogachevskii, N. Kleeorin and A. Brandenburg, J. Plasma Phys. 84, 735840502 (2018).

[8] F. Krause and K. H. Rädler, Mean-Field Magnetohydrodynamics and Dynamo Theory (Pergamon Press, Oxford, 1980).

[9] T. Elperin, N. Kleeorin and I. Rogachevskii, Phys. Rev. E 52, 2617 (1995).

[10] T. Elperin, N. Kleeorin and I. Rogachevskii, Phys. Rev. Lett. 76, 224 (1996).

[11] T. Elperin, N. Kleeorin and I. Rogachevskii, Phys. Rev. E 55, 2713 (1997).

[12] N. Yokoi, J. Plasma Phys. 84, 735840501 (2018).

[13] N. Yokoi, J. Plasma Phys. 84, 775840603 (2018).

[14] T. Elperin, N. Kleeorin, I. Rogachevskii and D. Sokoloff, Phys. Rev. E 61, 2617 (2000).
[15] T. Elperin, N. Kleeorin, I. Rogachevskii and D. Sokoloff, Phys. Rev. E 64, 026304 (2001).

[16] R. V. R. Pandya and F. Mashayek, Phys. Rev. Lett. 88, 044501 (2002).

[17] M. W. Reeks, Int. J. Multiph. Flow 31, 93 (2005).

[18] G. Amir, N. Bar, A. Eidelman, T. Elperin, N. Kleeorin, and I. Rogachevskii, Phys. Rev. Fluids 2, 064605 (2017).

[19] N. E. L. Haugen, N. Kleeorin, I. Rogachevskii, A. Brandenburg, Phys. Fluids 24, 075106 (2012).

[20] A. Brandenburg, K. H. Rädler, K. Kemel, Astron. Astrophys. 539, A35 (2012).

[21] J. Buchholz, A. Eidelman, T. Elperin, G. Grünefeld, N. Kleeorin, A. Krein, I. Rogachevskii, Experim. Fluids 36, 879 (2004).

[22] A. Eidelman, T. Elperin, N. Kleeorin, I. Rogachevskii and I. Sapir-Katiraie, Experim. Fluids 40, 744 (2006).

[23] A. Eidelman, T. Elperin, N. Kleeorin, B. Melnik and I. Rogachevskii, Phys. Rev. E 81, 056313 (2010).

[24] M. Sofiev, V. Sofieva, T. Elperin, N. Kleeorin, I. Rogachevskii and S. S. Zilitinkevich, J. Geophys. Res. 114, D18209 (2009).

[25] A. Hubbard, Monthly Notes Roy. Astron. Soc. 456, 30793089 (2016).

[26] M. Caporaloni, F. Tampieri, F. Trombetti and O. Vittori, J. Atmosph. Sci. 32, 565 (1975).

[27] M. Reeks, J. Aerosol Sci. 14, 729 (1983).

[28] A. Guha, J. Aerosol Sci. 28, 1517 (1997), Annu. Rev. Fluid Mech. 40, 311 (2008).

[29] T. Elperin, N. Kleeorin and I. Rogachevskii, Int. J. Multiphase Flow 24, 1163 (1998).

[30] Dh. Mitra, N. E. L. Haugen and I. Rogachevskii, Europ. Phys. J. Plus 133, 35 (2018). 
[31] S. I. Braginsky and P. H. Roberts, Geophys. Astrophys. Fluid Dyn. 79, 1 (1995).

[32] I. Rogachevskii and N. Kleeorin, J. Plasma Phys. 81, 395810504 (2015).

[33] T. Elperin, N. Kleeorin and I. Rogachevskii, Phys. Rev. E 55, 7043 (1997).

[34] L. D. Landau and E. M. Lifshits, Fluid Mechanics (Pergamon, Oxford, 1987).

[35] S. Chandrasekhar, Rev. Mod. Phys. 15, 1 (1943).

[36] A. I. Akhiezer and S. V. Peletminsky, Methods of Statistical Physics (Pergamon, Oxford, 1981).

[37] A. Favre, Statistical equations of turbulent gases, in: Problems of Hydrodynamic and Continuum Mechanics, SIAM, Philadelphia, 1969, pp. 231-266.

[38] S. K. Lele, Annu. Rev. Fluid Mech. 26, 211 (1994).

[39] W. D. McComb, The Physics of Fluid Turbulence (Clarendon, Oxford, 1990).

[40] U. Frisch, Turbulence: the Legacy of A. N. Kolmogorov (Cambridge University Press, Cambridge, 1995).

[41] S. B. Pope, Turbulent flows (Cambridge Univ. Press, Cambridge, 2000).

[42] P. H. Roberts and A. M. Soward, Astron. Nachr. 296, 49 (1975).

[43] G. K. Batchelor, The Theory of Homogeneous Turbulence (Cambridge University Press, Cambridge, 1953).

[44] S. A. Orszag, J. Fluid Mech. 41, 363 (1970).

[45] A. Pouquet, U. Frisch, and J. Leorat, J. Fluid Mech. 77, 321 (1976).

[46] N. Kleeorin, I. Rogachevskii, and A. Ruzmaikin, Zh. Eksp. Teor. Fiz. 97, 1555 (1990) [Sov. Phys. JETP 70, 878 (1990)].

[47] A. Brandenburg and K. Subramanian, Phys. Rept. 417, 1 (2005).

[48] A. Brandenburg, P. Käpylä, and A. Mohammed, Phys. Fluids 16, 1020 (2004).

[49] A. Brandenburg and K. Subramanian, Astron. Astrophys. 439, 835 (2005).

[50] A. Brandenburg and K. Subramanian, Astron. Nachr. 326, 400 (2005).

[51] A. Brandenburg, K.-H. Rädler, M. Rheinhardt, P. J.
Käpylä, Astrophys. J. 676, 740 (2008).

[52] I. Rogachevskii, N. Kleeorin, P. J. Käpylä, A. Brandenburg, Phys. Rev. E 84, 056314 (2011).

[53] T. Elperin, N. Kleeorin, M. Liberman, A. N. Lipatnikov, I. Rogachevskii, R. Yu, Phys. Rev. E 96, 053111 (2017).

[54] P. Chassaing, R. A. Antonia, F. Anselmet, L. Joly and S. Sarkar, Variable Density Fluid Turbulence (Springer Science \& Business Media, 2013), v. 69.

[55] A. G. Kritsuk, M. L. Norman, P. Padoan, and R. Wagner, Astrophys. J. 665, 416 (2007).

[56] C. Federrath, Mon. Not. R. Astron. Soc. 436, 1245 (2013).

[57] H. Aluie, Physica D 247, 54 (2013).

[58] S. Pan and E. Johnsen, J. Fluid Mech. 833, 717 (2017).

[59] M. J. Lighthill, Proc. Roy. Soc. A211, No. 1107, 564 (1952); Proc. Roy. Soc. A222, No. 1148, 1 (1954).

[60] I. Proudman, Proc. Roy. Soc. A214, No. 1116, 119 (1952).

[61] E. G. Blackman and G. B. Field, Phys. Fluids 15, L73 (2003).

[62] T. Elperin, N. Kleeorin, I. Rogachevskii and S.S. Zilitinkevich, Phys. Rev. E 66, 066305 (2002); BoundaryLayer Meteorol. 119, 449 (2006).

[63] J. L. Lumley, Phys. Fluids, 101405 (1967); J. C. Wyngaard and O. R. Cote, Q. J. R. Meteorol. Soc. 98, 590 (1972); S. G. Saddoughi and S. V. Veeravalli, J. Fluid Mech. 268, 333 (1994); T. Ishihara, K. Yoshida and Y. Kaneda, Phys. Rev. Lett. 88, 154501 (2002).

[64] S. S. Zilitinkevich, Turbulent Penetrative Convection (Avebury Technical, Aldershot, 1991); J. C. Kaimal and J. J. Fennigan, Atmospheric Boundary Layer Flows (Oxford University Press, New York, 1994); E. B. Kraus and J. A. Businger, Atmosphere-Ocean Interaction (Oxford University Press, Oxford, 1994).

[65] I. Rogachevskii and N. Kleeorin, Phys. Rev. E 61, 5202 (2000); 64, 056307 (2001); 70, 046310 (2004).

[66] K.-H. Rädler, N. Kleeorin and I. Rogachevskii, Geophys. Astrophys. Fluid Dyn. 97, 249 (2003); N. Kleeorin and I. Rogachevskii, Phys. Rev. E 67, 026321 (2003). 